\title{
Coenzyme Q10 supplementation for diabetes and its complications: an overview
}

\author{
DAVID MANTLE
}

\begin{abstract}
This article reviews the potential role of dietary supplementation with coenzyme Q10 (CoQ10) for the management of patients with type 2 diabetes. The rationale for supplementation with CoQ10 is based on its key roles in cellular energy metabolism and as an antioxidant, and its potential mediation of oxidative stress and inflammation, which have been implicated in the pathogenesis of diabetes. Randomised controlled clinical trials have demonstrated that supplementation with CoQ10 can significantly improve glycaemic control, as well as improving vascular dysfunction. Supplementation with CoQ10 may be of particular importance in type 2 diabetics prescribed statins and in those with fatty liver disease. Supplemental CoQ10 is usually well tolerated, with no significant adverse effects reported in long-term use. The importance of product quality and bioavailability cannot be over-emphasised, since this may be a factor in the disparity of findings from clinical trials supplementing CoQ10.

Br J Diabetes 2017:17:145-148
\end{abstract}

Key words: coenzyme Q10, diabetes, glycaemic control, vascular dysfunction, statins, fatty liver disease

\section{Introduction}

This article focuses principally on the potential role of dietary supplementation with coenzyme Q10 (CoQ10) in type 2 diabetes in human subjects, both with respect to the primary defect of compromised glycaemic control and the secondary consequences relating to vascular dysfunction and organ damage. The link between CoQ10, diabetes and fatty liver disease is also discussed. Although work has been carried out to investigate the action of supplementary CoQ10 in animal models of diabetes ${ }^{1-3}$ and in uncontrolled human studies, ${ }^{4}$ this article is concerned with recent randomised controlled clinical trials unless otherwise indicated.

\section{Coenzyme Q10 (CoQ10)}

CoQ10 is a vitamin-like substance which plays a key role in the

Pharma Nord (UK) Ltd, Morpeth, Northumberland, UK

Address for correspondence: Dr David Mantle Pharma Nord (UK) Ltd, Telford Court, Morpeth, Northumberland NE61 2DB, UK

Tel: +44 (0)1670 534900

E-mail:dmantle@pharmanord.co.uk

https://doi.org/10.15277/bjd.2017.149 biochemical process that supplies all cells with the energy required for their normal functioning; this is of particular importance in tissues with a high energy requirement, such as the heart. CoQ10 is also important as a lipid soluble antioxidant, protecting cells from the potentially damaging effects of toxic free radical species generated during normal cellular metabolism (oxidative stress).

CoQ10 occurs within the body in two very closely related chemical forms, an oxidised form (ubiquinone) and a reduced form (ubiquinol). The chemical structure of CoQ10 is relatively complex, and ubquinol differs from ubiquinone only by the addition of an extra two hydrogen atoms. Some supplement manufacturers claim that ubiquinol is the more important bioactive form of CoQ10; this is incorrect because, although CoQ10 is transported in blood in the reduced ubiquinol form, it is continually interconverted between oxidised and reduced forms within cells, and thus ubiquinone and ubiquinol CoQ10 forms are of equal metabolic importance.

Most of the body's daily CoQ10 requirement is synthesised endogenously, with a relatively small proportion obtained from the normal diet. Based on a total body pool of $2 \mathrm{~g}$ and average tissue turnover time of 4 days, the requirement for CoQ10 has been estimated at $500 \mathrm{mg} /$ day. A relatively small proportion of this daily requirement is obtained from the normal diet, typically up to $5 \mathrm{mg} /$ day. ${ }^{5}$ As people age, the capacity of the body to produce CoQ10 declines continuously; optimal production occurs around the midtwenties, with a continual decline thereafter (by approximately $50 \%$ at age 60 ).

An adequate supply of CoQ10 is essential for normal functioning of mitochondria (the subcellular organelles present in all cells where energy generation takes place), and CoQ10 depletion, oxidative stress and mitochondrial dysfunction have been implicated in the pathogenesis of diabetes. ${ }^{6,7}$

\section{Effect of CoQ10 on blood glucose control}

Several studies have reported significantly reduced blood CoQ10 levels in type 2 diabetic patients, correlating with increased levels of plasma glucose, $\mathrm{HbA}_{1 \mathrm{c}}$ and markers of oxidative stress. ${ }^{8}$ It has been proposed that, in older patients with type 2 diabetes (in whom blood CoQ10 levels have become depleted to $0.35 \mu \mathrm{g} / \mathrm{ml}$ or less) and who have become refractory to hypoglycaemic drugs, supplementation with CoQ10 is most effective; in particular, the subsequent response to hypoglycaemic drugs is significantly improved (W Judy, unpublished data).

Earlier randomised controlled clinical studies to determine the effect of CoQ10 supplementation on glycaemic control in type 2 diabetics gave mixed results. Thus Eriksson et a ${ }^{9}$ reported that supplementation with CoQ10 (200 mg/day for 6 months) did not affect 
glycaemic control whilst Hodgson et a/10 found CoQ 10 supplementation (200 mg/day for 3 months) significantly improved long-term glycaemic control (mean $\mathrm{HbA}_{1 c}$ reduced by $0.4 \%$ vs. placebo) and blood pressure (reductions in mean systolic blood pressure of 6 $\mathrm{mmHg}$ and diastolic blood pressure of $3 \mathrm{mmHg}$, respectively, vs. placebo)

In more recent randomised controlled studies, KolahdouzMohammadi et a ${ }^{111}$ reported CoQ10 supplementation (200 mg/day for 3 months) significantly reduced $\mathrm{HbA}_{1 \mathrm{c}}$ levels (mean $\mathrm{HbA}_{1 \mathrm{c}}$ reduced by $0.6 \%$ vs. placebo) in type 2 diabetics. Similarly, Zahedi et $\mathrm{a}^{12}$ found that CoQ10 supplementation $(150 \mathrm{mg} /$ day for 3 months) significantly improved fasting plasma glucose (mean FPG reduced by $2.05 \mathrm{mmol} / \mathrm{L}\left(37 \mathrm{mg} / \mathrm{dL}\right.$ ) vs. placebo) and $\mathrm{HbA}_{1 \mathrm{c}}$ levels (mean $\mathrm{HbA}_{1 c}$ reduced by $0.8 \%$ vs. placebo), and HosseinzadehAttar et a ${ }^{13}$ reported significant improvement in $\mathrm{HbA}_{1 c}$ levels (mean $\mathrm{HbA}_{1 \mathrm{c}}$ reduced by $0.5 \%$ vs. placebo) following supplementation with $200 \mathrm{mg} /$ day for 3 months. In type 2 diabetic patients with neuropathy, supplementation with CoQ10 (200 mg/day for 3 months) did not significantly benefit neuropathic symptoms, $\mathrm{HbA}_{1 \mathrm{c}}$ or fasting glucose levels, but reduced inflammation and increased insulin sensitivity. ${ }^{14}$ Serum insulin and glucose homeostasis parameters were significantly improved in patients with metabolic syndrome/ type 2 diabetes following CoQ10 supplementation $(100 \mathrm{mg} /$ day for 2 months)..$^{15}$ In overweight or obese patients with type 2 diabetes, supplementation with CoQ10 (200 mg/day for 3 months) significantly reduced $\mathrm{HbA}_{1 c}$ levels (mean baseline $\mathrm{HbA}_{1 c}$ reduced by $0.7 \%$ ) and decreased weight and waist circumference. ${ }^{16}$

The question arises as to how CoQ10 might directly influence glycaemic homeostasis. In general terms, the metabolism of glucose involves a number of energy-dependent processes; if the body's energy supply mechanism is compromised via mitochondrial dysfunction/coenzyme Q10 deficiency, then it follows that glycaemic control must be affected. More specifically, CoQ10 has been shown to mediate directly the expression of over 100 genes involved in cellular metabolism, and data from diabetic animal models have shown CoQ10 can modulate components of the insulin signalling pathway (insulin receptors, tyrosine kinase and phosphatidylinositol kinase), glucose metabolism (glucose transporters, adiponectin) and oxidative stress (malondialdehyde). ${ }^{17}$

\section{Effect of CoQ10 supplementation on vascular dysfunction}

Type 2 diabetes is characterised by increased risk of damage to blood vessels throughout the body, resulting in the long term in ulceration of the feet and lower legs, renal failure, heart failure and nerve damage. For example, diabetic retinopathy typically develops in some $60 \%$ of type 2 diabetics over a period of approximately 20 years. Increased blood glucose levels cause glycation of vessel wall proteins resulting in endothelial cell dysfunction, as well as changes in erythrocyte aggregation/adhesion characteristics. Damage to blood vessels is linked to free radicalinduced damage resulting from diabetic-related oxidative stress and its associated inflammation.

Increased oxidative stress in type 2 diabetes correlates with reduced CoQ10 levels. ${ }^{18}$ Markers of oxidative stress, including oxidised LDL cholesterol and the redox regulating protein glutaredoxin, were significantly improved in patients with type 1 and type 2 diabetes following CoQ10 supplementation (200 mg/day for 3 months). ${ }^{19}$ Supplementation with CoQ10 (200 mg/day for 3 months) in type 2 diabetics significantly improved endothelial dysfunction, quantified via brachial artery flow-mediated dilation, translating as a $10-20 \%$ reduction in residual cardiovascular risk. ${ }^{20}$ Hosseinzadeh-Attar et a ${ }^{/ 13}$ found CoQ10 supplementation (200 $\mathrm{mg} /$ day for 3 months) significantly reduced circulatory levels of asymmetric dimethylarginine, a marker of endothelial dysfunction and cardiovascular event risk factor. In the Q-SYMBIO study (a randomised double-blind multicentre trial of CoQ10 as adjunctive treatment of chronic heart failure with focus on SYMptoms, BIOmarker status and long-term outcome), supplementation with CoQ10 (300 mg/day for 2 years) improved heart function and reduced cardiovascular-related mortality by $43 \%$ in heart failure patients, $10 \%$ of whom had type 2 diabetes. ${ }^{21}$ Similarly, in the KISEL-10 study (a 5-year prospective randomised double-blind placebo-controlled trial of the effect of intervention with selenium and CoQ10 on cardiovascular mortality and cardiac function in the elderly population in Sweden), supplementation with 200 mg/day CoQ10 and $200 \mu \mathrm{g} /$ day selenium for 5 years reduced cardiovascular mortality by $53 \%$ in elderly subjects, $20 \%$ of whom had diabetes. ${ }^{22}$

\section{CoQ10, statins and diabetes}

It is generally recognised that there is an increased risk (depending on a number of factors) of approximately $10 \%$ of developing type 2 diabetes in patients prescribed statins (particularly simvastatin). ${ }^{23}$ However, Cederberg et a ${ }^{R^{4}}$ have recently quoted an increased risk of $46 \%$ of developing type 2 diabetes in patients prescribed statins (simvastatin, atorvastatin), resulting from decreased insulin secretion/sensitivity. It is of note that simvastatin and atorvastatin are the two statin types which induce the greatest reductions in CoQ10 synthesis within the body; as well as reducing endogenous synthesis of cholesterol, statins also inhibit the production of CoQ10 which is synthesised via the same biochemical pathway. Statin-induced diabetes has been linked to reductions in circulatory adiponectin and CoQ10 levels, and a reduction in the protein glucose transporter type 4 (GLUT4), which helps insulin to control blood glucose levels. ${ }^{25}$ In cell culture, administration of CoQ10 prevents the reduction in GLUT4 protein levels caused by simvastatin, ${ }^{26}$ although these findings need to be confirmed in human subjects.

\section{CoQ10, diabetes and liver disease}

Non-alcoholic fatty liver disease (NAFLD) is the most common liver disorder worldwide, with a prevalence in the general population estimated at 20-30\%. Estimation of the incidence of NAFLD in diabetes varies with the population studied and analytical method used (biochemical, histological, ultrasonography or magnetic resonance imaging). In those with type 2 diabetes, this has been estimated by some authorities to be as high as $70 \%$, rising to $90 \%$ in those with morbid obesity. 27,28

Although CoQ10 is synthesised throughout the body, because of its size and high metabolic capacity, the liver is the major site of CoQ10 synthesis. In patients with liver disease 
where metabolic capacity has been compromised, reduction in CoQ10 production is likely to have a deleterious effect on heart function. Thus, NAFLD is a risk factor for cardiovascular disease, which has been reported to be one of the major causes of death in NAFLD patients. ${ }^{29}$ NAFLD is associated with heart failure, arrhythmias, valve dysfunction and atherosclerosis. ${ }^{30}$ Reduced CoQ10 levels may be a particular problem in patients with fatty liver disease prescribed statins since, in addition to inhibiting cholesterol synthesis, statins also inhibit the production of CoQ10.

In addition to its role in cellular energy production and as an antioxidant, CoQ10 has been shown to directly influence the expression of a number of genes, including those involved in inflammation and oxidative stress. . $^{31,32}$ Thus, as well as reducing the risk of cardiovascular problems in patients with liver disease, CoQ10 supplementation may also benefit the disease process within the liver by reducing inflammation and oxidative stress, key factors in the pathogenesis of NAFLD. ${ }^{33}$

Yesilova et $a^{\beta 4}$ found that blood CoQ10 levels were depleted in NAFLD patients, with the decrease in CoQ10 correlating with increased liver inflammation and cirrhosis. A randomised controlled trial by Farhangi et a ${ }^{\beta 5}$ found supplementation with CoQ10 (100 mg/day for 4 weeks) resulted in decreased systemic levels of biochemical markers of inflammation and oxidative stress. In a more recent randomised controlled trial, ${ }^{36}$ NAFLD patients given $100 \mathrm{mg}$ CoQ10 per day for 3 months showed significant reductions in the levels of blood markers for liver inflammation and damage (aspartate aminotransferase, gammaglutamyl peptidase, C-reactive protein).

It follows from the above that diabetes, NAFLD and cardiovascular disease are interlinked, with deficiency of CoQ10 as a common denominator.

\section{Safety of CoQ10}

CoQ10 is generally well tolerated, with no serious adverse effects reported in long-term use. Very rarely, individuals may experience mild gastrointestinal disturbance. There are no known toxic effects, and CoQ10 cannot be overdosed. ${ }^{37,38}$ The safety of CoQ10 has been confirmed in more than 200 randomised controlled trials in a wide range of disorders. Several case studies have suggested that CoQ10 may interfere with the action of warfarin; however, a randomised controlled clinical trial showed CoQ10 supplementation at $100 \mathrm{mg} /$ day had no effect on the clinical action of warfarin. ${ }^{39}$

Because food supplements are not regulated in the same way as prescription medicines, concerns have been raised regarding the potential variability of various CoQ10 supplements used in clinical trials; in particular, there is a common misconception amongst healthcare professionals that there are no regulatory agency approvals for CoQ10. ${ }^{40}$ This is incorrect, as one CoQ10 product has a Marketing Authorisation within the EU for the adjuvant treatment of chronic heart failure. As part of the licensing procedure, periodic safety update reports (PSURs) have to be submitted every 3 years. In a sample PSUR, over a 3-year period the supply of 1.4 million daily doses, equivalent to 3,900 person-years usage, did not result in any reported serious adverse events ascribable to CoQ10.

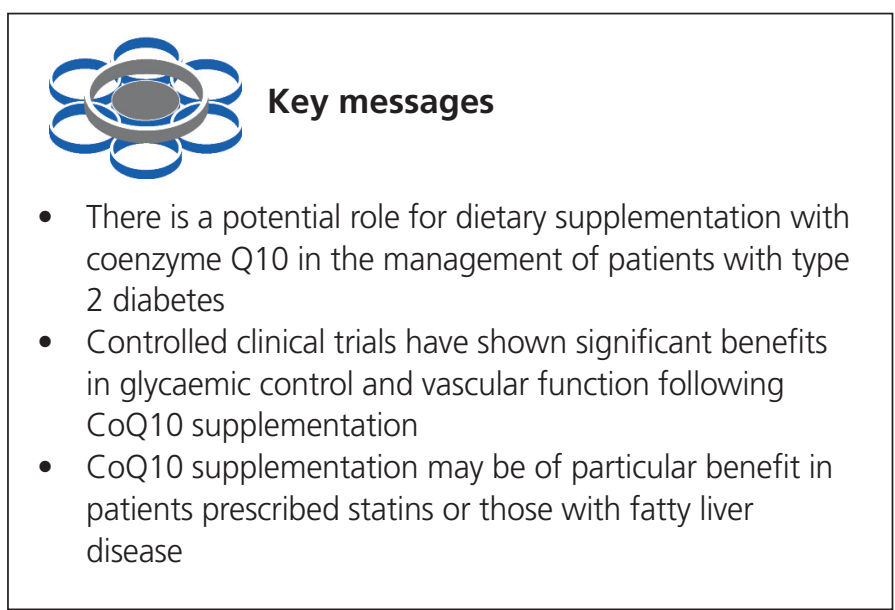

CoQ10: importance of product quality and bioavailability Bioavailability is defined as the proportion of an ingested substance that reaches the blood circulation. Because of its relatively large molecular size and lipid solubility, the bioavailability of CoQ10 is intrinsically low. CoQ10 is absorbed from the intestinal tract by the same mechanism as other lipid soluble nutrients, via a lipid carrier through mucosal cells initially into the lymph, and thence into the bloodstream; thus absorption is optimised when CoQ10 is dissolved in a carrier oil (preferably soya or palm oil). The delayed peak concentration of CoQ10 in blood is a consequence of this initial absorption into the lymphatic system. However, incorporation of supplemental CoQ10 in liposomal, micellar or nanoparticle form cannot increase the absorption of CoQ10 through intestinal cells into the lymph. CoQ10 cannot be made more water soluble, as any alteration to the molecular structure means that the molecule is no longer CoQ10. When supplemental CoQ10 is first produced (via a yeast fermentation process), it is obtained in the form of crystals which cannot be absorbed from the digestive tract. It is essential that these crystals are dispersed into single CoQ10 molecules (and remain dispersed during the product shelf-life) to enable optimum bioavailability; adding CoQ10 crystals to a carrier oil without such dispersal, a cost-saving technique used by some manufacturers, is inadequate. Disparity in the findings of clinical trials supplementing CoQ10 in diabetes may result from a number of factors, including inadequate bioavailability and insufficient dosage or treatment duration, as well as inter-individual variation in the ability to absorb CoQ10.

Conflict of interest DM is medical adviser to Pharma Nord (UK) Ltd a manufacturer of $\mathrm{COQ} 10$ supplements.

Funding None.

\section{References}

1. De Blasio MJ, Huynh K, Qin C, et al. Therapeutic targeting of oxidative stress with CoQ10 counteracts exaggerated diabetic cardiomyopathy in a mouse model of diabetes with diminished PI3K(p110 $\alpha)$ signaling. Free Radic Biol Med 2015:87:137-47.

http://dx.doi.org/10.1016/j.freeradbiomed.2015.04.028

2. Mao Z, Wu JH, Dong T, Wu MX. Additive enhancement of wound healing in diabetic mice by low level light and topical CoQ10. Sci Rep 2016:6:20084. http://dx.doi.org/10.1038/srep20084 
3. Zhang YP, Mei S, Yang J, et al. Acute hypoglycemia induces painful neuropathy and the treatment of CoQ10. J Diabetes Res 2016:4593052. http://dx.doi.org/10.1155/2016/4593052

4. Mezawa M, Takemoto M, Onishi S, et al. The reduced form of CoQ10 improves glycemic control in patients with type 2 diabetes: an open label study. Biofactors 2012;38:416-21. http://dx.doi.org/10.1002/biof.1038.

5. Weber C, Bysted A, Hilmer G. The CoQ10 content of the average Danish diet. Int J Vitam Nutr Res 1997:67:123-9.

6. Petersen KF, Befroy D, Dufour S. Mitochondrial dysfunction in the elderly: possible role in insulin resistance. Science 2003;300:1140-2. http://dx.doi.org/10.1126/science.1082889

7. Naudi A, Jove M, Ayala V, et al. Cellular dysfunction in diabetes as maladaptive response to mitochondrial oxidative stress. Exp Diabetes Res 2012:696215. http://dx.doi.org/ 10.1155/2012/696215

8. El-Ghoroury EA, Raslan HM, Badawy EA, et al. Malondialdehyde and CoQ10 in platelets and serum in type II diabetes: correlation with glycemic control. Blood Coagul Fibrinolysis 2009;20:248-51. https://doi.org/10.1097/MBC.0b013e3283254549

9. Eriksson JG, Forsen TJ, Mortensen SA, Rohde M. Effect of CoQ10 administration on metabolic control in patients with type 2 diabetes mellitus. Biofactors 1999:9:315-18. https://doi.org/10.1002/biof.5520090229

10. Hodgson JM, Watts GF, Playford DA, Burke V, Croft KD. CoQ10 improves blood pressure and glycaemic control in subjects with type 2 diabetes. Eur J Clin Nutr 2002;56:1137-42. http://dx.doi.org/10.1038/sj.ejcn.1601464

11. Kolahdouz-Mohammadi R, Hosseinzadeh-Attar M, Eshraghian M, et al. Effect of CoQ10 supplementation on metabolic status of type 2 diabetic patients. Minerva Gastroenterol Dietol 2013;59:231-5.

12. Zahedi H, Eghtesadi S, Seifirad S, et al. Effects of CoQ10 supplementation on lipid profiles and glycemic control in patients with type 2 diabetes. J Diabetes Metab Disord 2014;13:81-9. http://dx.doi.org/10.1186/s40200-014-0081-6

13. Hosseinzadeh-Attar M, Mohammadi R, Eshraghian IM, et al. Reduction in asymmetric dimethylarginine plasma levels by CoQ10 supplementation in patients with type 2 diabetes. Minerva Endocrinol 2015;40:25966 .

14. Akbari-Fakhrabadi M, Ghotrom A, Khosravi H, et al. Effect of CoQ10 on oxidative stress, glycemic control and inflammation in diabetic neuropathy: a double blind randomised clinical trial. Int J Vitam Nutr Res 2014;84:252-60. http://dx.doi.org/10.1024/0300-9831/a000211

15. Raygan F, Rezawandi Z, Dadkhah Tehrani S, Farrokhian A, Asemi Z. The effects of CoQ10 administration on glucose homeostasis parameters, lipid profiles, biomarkers of inflammation and oxidative stress in patients with metabolic syndrome. Eur J Nutr 2015;55:2357-64. http://dx.doi.org/10.1007/s00394-015-1042-7

16. Mehrdadi P, Mohammadi R, Alipoor E, et al. Effect of CoQ10 supplementation on circulating levels of adipokine adipolin in overweight and obese patients with type 2 diabetes. Exp Clin Endocrinol Diabetes 2016:125:156-62. http://dx.doi.org/10.1055/s-0042-110570

17. Amin MM, Asaad GF, Salam RF, El-Abhar HS, Arbid MS. Novel CoQ10 antidiabetic mechanisms underlie its positive effect: modulation of insulin and adiponectin receptors, tyrosine kinase, glucose transporters and visfatin in insulin resistant/diabetic rats. PloS One 2014;9:e89169. http://dx.doi.org/10.1371/journal.pone.0089169

18. Ates $\mathrm{O}$, Bileu $\mathrm{H}$, Keies $\mathrm{S}$, et al. Plasma CoQ10 levels in type 2 diabetics with retinopathy. Int J Ophthalmol 2013;6:675-9. http://dx.doi.org/10.3980/j.issn.2222-3959.2013.05.24

19. Montano SJ, Grinier J, Nair D, et al. Glutaredoxin mediated redox effects of CoQ10 treatment in type 1 and type 2 diabetes patients. BBA Clin 2015;4:14-20. http://dx.doi.org/10.1016/j.bbacli.2015.06.001

20. Hamilton SJ, Chew GT, Watts GF. CoQ10 improves endothelial dysfunction in statin treated type 2 diabetic patients. Diabetes Care 2009; 32:810-12. http://dx.doi.org/10.2337/dc08-1736

21. Mortensen SA, Rosenfeldt F, Kumar A, et al. The effect of CoQ10 on morbidity and mortality in chronic heart failure: results from Q-SYMBIO: a randomised double blind trial. JACC Heart Fail 2014;2:641-9. http://dx.doi.org/10.1016/j.jchf.2014.06.008

22. Alehagen $U$, Johansson $P$, Bjornstadt $M$, Rosén $A$, Dahlström U. Cardiovascular mortality and $\mathrm{N}$-terminal-proBNP reduced after combined se- lenium and CoQ10 supplementation: a 5 year prospective randomised double blind placebo controlled trial among elderly Swedish citizens. J Cardio/ 2013;167:1860-6. http://dx.doi.org/10.1016/j.ijcard.2012.04.156

23. Brault M, Ray J, Gomez YH, Mantzoros CS, Daskalopoulou SS. Statin treatment and new onset diabetes: a review of proposed mechanisms. Metabolism 2014:63:735-45. http://dx.doi.org/10.1016/j.metabol.2014.02.014

24. Cederberg H, Stančáková A, Yaluri N, Modi S, Kuusisto J, Laakso M. Increased risk of diabetes with statin treatment is associated with impaired insulin sensitivity and insulin secretion: a 6 year follow up study of the METSIM cohort. Diabetologia 2015;58:1109-17. http://dx.doi.org/10.1007/s00125-015-3528-5

25. Chan DC, Pang J, Watts DF. Pathogenesis and management of the diabetogenic effect of statins: a role for adiponectin and CoQ10? Curr Atheroscler Rep 2015;17:472-5. http://dx.doi.org/10.1007/s11883-014-0472-7

26. Gansean S, Ito MK. CoQ10 ameliorates reduction in GLUT4 transporter expression induced by simvastatin in 3T3-L1 adipocytes. Metab Syndr Relat Disord 2013;11:251-5. http://dx.doi.org/10.1089/met.2012.0177

27. Ahmed M. Non-alcoholic fatty liver disease in 2015. World J Hepatol 2015;7:1450-9. http://dx.doi.org/10.4254/wjh.v7.i11.1450

28. Hazelhurst JM, Woods C, Marjot T, Cobbold JF, Tomlinson JW. Nonalcoholic fatty liver disease and diabetes. Metabolism 2016;65:1096108. http://dx.doi.org/10.1016/j.metabol.2016.01.001

29. Francque SM, Graff D, Kwantan WJ. NAFLD and cardiovascular risk: pathophysiological mechanisms and implications. J Hepatol 2016;65 425-43. http://dx.doi.org/10.1016/j.jhep.2016.04.005

30. Sirbu O, Florida M, Dascalita P, Sorodoc V, Sorodoc L. NAFLD from the cardiologist perspective. Anatol J Cardiol 2016;16:534-41. http://dx.doi.org/10.14744/AnatolJCardiol.2016.7049

31. Lee BJ, Tseng YF, Yen CH, Lin PT. Effects of CoQ10 supplementation (300 mg/day) on antioxidation and anti-inflammation in coronary artery disease patients during statins therapy: a randomized, placebo-controlled trial. Nutr J 2013;12:142-8. http://dx.doi.org/10.1186/1475-2891-12-142

32. Fan L, Feng Y, Chen GC, Qin LQ, Fu CL, Chen LH. Effects of CoQ10 supplementation on inflammatory markers: a systematic review and metaanalysis of randomised controlled trials. Pharmacol Res 2017;119: 128-36. http://dx.doi.org/10.1016/j.phrs.2017.01.032

33. Spahis S, Delvin E, Borys J, Levy E. Oxidative stress as a critical factor in NAFLD. Antioxid Redox Signal 2017;26:519-41. http://dx.doi.org/10.1089/ars.2016.6776

34. Yesilova Z, Yaman H, Oktenli C, et al. Systemic markers of lipid peroxidation and antioxidants in patients with non-alcoholic fatty liver disease. Am J Gastroenterol 2005;100:850-5.

http://dx.doi.org/10.1111/j.1572-0241.2005.41500.x

35. Farhangi MA, Alipour B, Jafarvand E, Khoshbaten M. Oral CoQ10 supplementation in patients with NAFLD: effects on serum vaspin, chemerin, pentraxin, insulin resistance and oxidative stress. Arch Med Res 2014;45:589-95. http://dx.doi.org/10.1016/j.arcmed.2014.11.001

36. Farsi F, Mohammadshalin M, Aalavinejad P, Rezazadeh A, Zarei M, Engal KA. Functions of CoQ10 supplementation on liver enzymes, markers of inflammation and adipokines in patients with NAFLD: a double blind randomized placebo controlled clinical trial. J Am Coll Nutr 2016; 35:346-53. http://dx.doi.org/10.1080/07315724.2015.1021057

37. Hosoe K, Kitano M, Kishida H, Kubo H, Fujii K, Kitahara M. Study on the safety and bioavailability of ubiquinol after single and 4-week multiple oral administration to healthy volunteers. Regul Toxicol Pharmacol 2007;47:19-28. http://dx.doi.org/10.1016/j.yrtph.2006.07.001

38. Hidaka T, Fujii K, Funahashi I, Fukutomi N, Hosoe K. Safety assessment of CoQ10. Biofactors 2008:32:199-208. https://doi.org/10.1002/biof.5520320124

39. Engelsen J, Nielsen JD, Hansen KF. Effect of CoQ10 and Ginkgo biloba on warfarin dosage in patients on long term warfarin treatment. A double blind placebo controlled cross-over trial. Ugeaskr Laeger 2003:165:1868-71.

40. Ezekowitz JA. Time to energise CoQ10 for patients with heart failure? JACC Heart Fail 2014;2:650-2. http://dx.doi.org/10.1016/j.jchf.2014.07.007 